\title{
新型膦胺配体羰基钉化合物的合成及其催化性能研究
}

\author{
方霄龙 ${ }^{a, b}$ 章 敏 ${ }^{a}$ 段 宁 ${ }^{a}$ 汪 新*,a 朱平*,b \\ ( ${ }^{a}$ 池州学院化学与材料工程学院 微纳粉体与先进能源材料安徽普通高校重点实验室 安徽池州 247000) \\ ( ${ }^{b}$ 厦门大学化学化工学院 固体表面物理化学国家重点实验室 醇醚酯清洁生产国家工程实验室 福建厦门 361005)
}

\begin{abstract}
摘要 以十二崤基三钓和 $o-\mathrm{PPh}_{2} \mathrm{C}_{6} \mathrm{H}_{4} \mathrm{NR}_{2}(\mathrm{R}=\mathrm{H}, \mathrm{Me})$ 配体为原料, 成功制备了三种新型羰基钓化合物 $\left(\mu-o-\mathrm{PPh}_{2}-\right.$ $\left.\mathrm{C}_{6} \mathrm{H}_{4} \mathrm{NH}\right) \mathrm{Ru}_{3}(\mu-\mathrm{H})(\mathrm{CO})_{9}(2) 、\left(o-\mathrm{PPh}_{2} \mathrm{C}_{6} \mathrm{H}_{4} \mathrm{NH}\right)_{2} \mathrm{Ru}(\mathrm{CO})_{2}(\mathbf{3})$ 和 $\left(\mu-o-\mathrm{PPh}_{2} \mathrm{C}_{6} \mathrm{H}_{4} \mathrm{NMe}_{2}\right)_{2} \mathrm{Ru}(\mathrm{CO})_{3}$ (4). 对这三个化合物进行了 核磁共振和红外谱学、元素分析和 X 射线单晶衍射分析表征, 并对这三个化合物进行了催化性能研究. 化合物 2 和 4 可催化苯甲醛加氢反应生成苯甲醇, 但是 $\mathbf{3}$ 没有催化活性. 从实验角度阐述了膦胺配体钓催化剂的结构与性能关联, 进一步探讨了加氢催化反应失活的可能原因.
\end{abstract}

关键词＼cjkstart膦胺配体羰基钉化合物; 合成与表征; 催化加氢; 结构与性能; 失活

\section{Synthesis and Catalytic Property of New Aminophosphino Ruthenium Carbonyl Complexes}

\author{
Fang, Xiaolong ${ }^{a, b} \quad{\text { Zhang, } \text { Min }^{a} \quad \text { Duan, Ning }}^{a} \quad$ Wang, Xin ${ }^{*, a} \quad$ Zhu, Hongping ${ }^{*, b}$ \\ ( ${ }^{a}$ Key Laboratory of Micro-nano Powder and Advanced Energy Materials of Anhui Higher Education Institutes, \\ College of Chemistry and Materials Engineering, Chizhou University, Chizhou, Anhui 247000) \\ $\left({ }^{b}\right.$ State Key Laboratory of Physical Chemistry of Solid Surfaces, National Engineering Laboratory for Green Chemical \\ Productions of Alcohols-Ethers-Esters, College of Chemistry and Chemical Engineering, \\ Xiamen University, Xiamen, Fujian 361005)
}

\begin{abstract}
New carbonyl ruthenium complexes $\left(\mu-o-\mathrm{PPh}_{2} \mathrm{C}_{6} \mathrm{H}_{4} \mathrm{NH}\right) \mathrm{Ru}_{3}(\mu-\mathrm{H})(\mathrm{CO})_{9}(\mathbf{2}),\left(o-\mathrm{PPh}_{2} \mathrm{C}_{6} \mathrm{H}_{4} \mathrm{NH}\right)_{2} \mathrm{Ru}(\mathrm{CO})_{2}(\mathbf{3})$ and $\left(\mu \text {-o- } \mathrm{PPh}_{2} \mathrm{C}_{6} \mathrm{H}_{4} \mathrm{NMe}_{2}\right)_{2} \mathrm{Ru}(\mathrm{CO})_{3}(4)$ have been successfully synthesized by using ruthenium carbonyl and $o-\mathrm{PPh}_{2} \mathrm{C}_{6} \mathrm{H}_{4} \mathrm{NR}_{2}(\mathrm{R}=$ $\mathrm{H}, \mathrm{Me}$ ) ligand. The three complexes have all been characterized by NMR and IR spectroscopies, elemental analysis and X-ray crystallography. Complexes $\mathbf{2}$ and $\mathbf{4}$ could catalyze the hydrogenation of benzaldehyde into benzyl alcohol. However, complex 3 showed no activity. This study reveals a correlation between structure and catalytic property, where the possible deactivation mode for the hydrogenation reaction using the aminophosphino ruthenium catalyst is discussed in view of the experimental work.

Keywords aminophosphino ruthenium carbonyl; synthesis and characterization; catalytic hydrogenation; structure and property; deactivation
\end{abstract}

在过渡金属化合物均相催化加氢反应中, 由于 $\mathrm{H}_{2}$ 分子的活化方式差异，目前基本上认为有两类反应机 理, 分别是金属-配体协同作用下的“金属配体双功能机 理” (metal-ligand bifunctional mechanism) 和金属中心作 用下的 “内核加氢机理” (inner-sphere hydrogenation mechanism ${ }^{[1]}$. 以含氮配体金属 $\mathrm{Ru}$ 化合物的催化反应 为例, $\mathrm{H}_{2}$ 分子易于与 $\mathrm{Ru}-\mathrm{NH}$ 结构单元作用转化生成
$\mathrm{H}_{2} \mathrm{~N} \rightarrow \mathrm{RuH}^{[2]}$ (或基于吡啶亚甲基配体的芳构化一去芳构 化过程, ligand aromatization-dearomatization process ${ }^{[3]}$ ). 在该反应中, $\mathrm{H}_{2}$ 分子发生的是异裂反应，其中金属 $\mathrm{Ru}$ 的 价态不发生变化; 生成的 $\mathrm{H}_{2} \mathrm{~N} \rightarrow \mathrm{RuH}$ 官能团用于催化反 应时, $\mathrm{Ru}-\mathrm{H}$ 与 $\mathrm{N}-\mathrm{H}$ 分别与底物分子通过协同的亲核 和亲电作用形成 “六元环” 过渡态, 并在 $\mathrm{Ru} \leftarrow \mathrm{N}$ 配位键 和 $\mathrm{Ru}-\mathrm{N}$ 共价键的转换过程中引发 $\mathrm{Ru}-\mathrm{H}$ 和 $\mathrm{N}-\mathrm{H}$ 对

* Corresponding authors. E-mail: wangxin164@sina.com; hpzhu@xmu.edu.cn

Received June 18, 2019; revised August 22, 2019; published online September 13, 2019.

Project supported by the National Natural Science Foundation of China (Nos. 21802010, 21673191), the Natural Science Foundation of Anhui Province (No. 1808085QB48) and the Starting Grants for Young Teachers of Chizhou University (No. 2018YJRC001).

国家自然科学基金(Nos. 21802010, 21673191)、安徽省自然科学基金(No. 1808085QB48)及池州学院青年教师科研启动基金(No. 2018YJRC001)资助项 目. 
底物分子中的 $\mathrm{C}=\mathrm{O}$ 键 $\mathrm{H}^{-} / \mathrm{H}^{+}$加成, 实现催化加氢 (Scheme 1a). 这是典型的 “金属配体双功能机理”。第 二种 “内核加氢机理” 是金属 $\mathrm{Ru}$ 通过氧化加成反应生 成 $\mathrm{RuH}_{2}{ }^{[4]}$. 在该反应中, $\mathrm{H}_{2}$ 分子均裂生成两个钉金属氢, 其中金属中心的价态由 0 价变为 +2 价. 在催化反应过 程中, 一个金属氢首先与底物分子的 $\mathrm{C}=\mathrm{O}$ 键进行加成 反应，生成的中间体进一步与另一个金属氢发生还原消 除反应，生成产物(Scheme 1b).

近年来, 我们课题组 ${ }^{[5]}$ 围绕 $o-\mathrm{PPh}_{2} \mathrm{C}_{6} \mathrm{H}_{4} \mathrm{NHR}(\mathrm{R}=\mathrm{H}$, $\mathrm{Me}, \mathrm{Et}, \mathrm{CH}_{2} \mathrm{Ph}$ )配体合成了系列不同结构的 $o-\mathrm{PPh}_{2} \mathrm{C}_{6} \mathrm{H}_{4}-$ $\mathrm{NHR}-\mathrm{Ru}$ 配合物, 并成功地将其应用于均相催化加氢反 应. 围绕催化活性物种 [如 $\left(o-\mathrm{PPh}_{2} \mathrm{C}_{6} \mathrm{H}_{4} \mathrm{NH}_{2}\right)\left(o-\mathrm{PPh}_{2} \mathrm{C}_{6} \mathrm{H}_{4}-\right.$ $\mathrm{NH}) \mathrm{RuH}(\mathrm{CO})$ (1) 等], 我们开展了相关的动力学以及密 度泛函理论计算研究. 结果证实了 $\mathrm{Ru}-\mathrm{H}$ 与 $\mathrm{N}-\mathrm{H}$ 的协 同作用过程 ${ }^{[5 a, 5 b]}$. 2018 年, 我们 ${ }^{[6]}$ 进一步将乙酰丙酮钌 和 $o-\mathrm{PPh}_{2} \mathrm{C}_{6} \mathrm{H}_{4} \mathrm{NH}_{2}$ 配体组成的催化剂体系应用于丙二酸 二甲酯加氢制 3-着基丙酸甲酯的催化反应. 发现溶剂对 催化活性有显著的影响. 在相同反应条件下, 采用甲

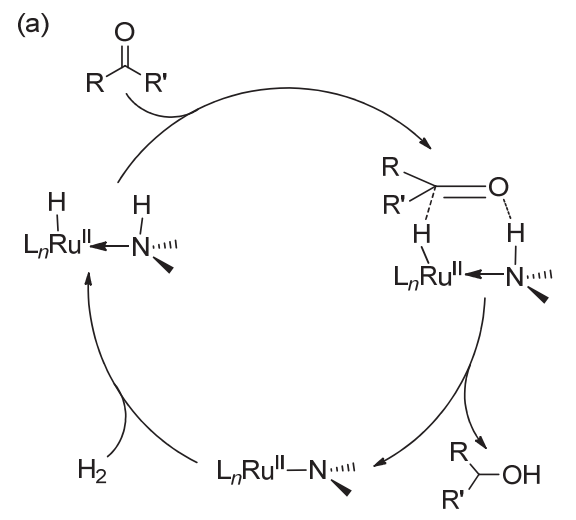

醇-四氢呋喃混合溶剂(体积比为 $2: 8$ )与仅采用甲醇作 为溶剂相比, 丙二酸二甲酯转化率以及 3 -差基丙酸甲酯 收率分别由 $55 \%$ 和 $45 \%$ 提升到 $86 \%$ 和 $75 \%$. 这一结果表 明采用混合溶剂可有效减缓反应过程中催化剂的失活 速率, 推测四氢呋喃(THF)是一种良好的电子给体, 一 定程度上稳定了金属活性中心，降低了甲醇分解产生 $\mathrm{CO}$ 毒化催化剂的进程. 在本研究中, 我们采用 $\mathrm{Ru}_{3}(\mathrm{CO})_{12}$ 与 $o-\mathrm{PPh}_{2} \mathrm{C}_{6} \mathrm{H}_{4} \mathrm{NR}_{2}(\mathrm{R}=\mathrm{H}, \mathrm{Me})$ 配体反应，成 功地合成了 3 种具有不同结构特征的羰基钉配合物 2 4, 初步考察了它们催化加氢的反应活性. 实验结果直 观地展示了 $\mathrm{Ru}-\mathrm{NH}$ 催化剂的协同作用原理，也探讨了 催化反应的失活机制.

\section{1 结果与讨论}

钌配合物 $2 \sim 4$ 的合成过程如 Scheme 2 所示. $\mathrm{Ru}_{3}(\mathrm{CO})_{12}$ 与 1 equiv. $o-\mathrm{PPh}_{2} \mathrm{C}_{6} \mathrm{H}_{4} \mathrm{NH}_{2}$ 的甲苯溶液在 $60{ }^{\circ} \mathrm{C}$ 条件下反应 $24 \mathrm{~h}$, 得到 $\left(\mu-o-\mathrm{PPh}_{2} \mathrm{C}_{6} \mathrm{H}_{4} \mathrm{NH}\right) \mathrm{Ru}_{3}(\mu-\mathrm{H})-$ $(\mathrm{CO})_{9}(2)$. 以化合物 2 为原料进一步与 5 equiv. $o-\mathrm{PPh}_{2}-$

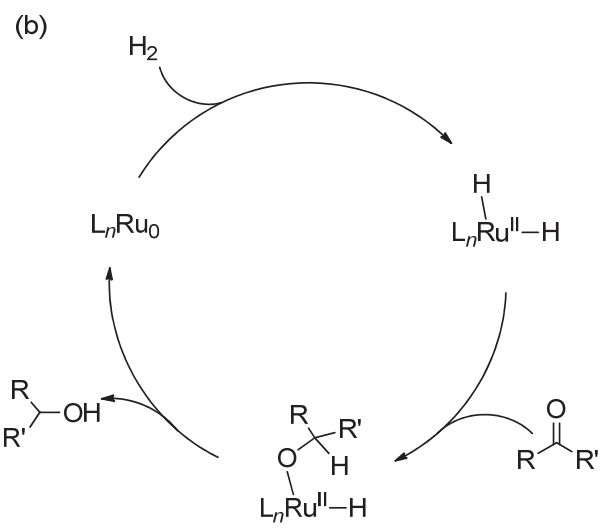

图式 1 钉化合物均相催化加氢的两种可能反应机理

Scheme 1 Two proposed reaction mechanisms for homogeneous hydrogenation catalyzed by the Ru complex

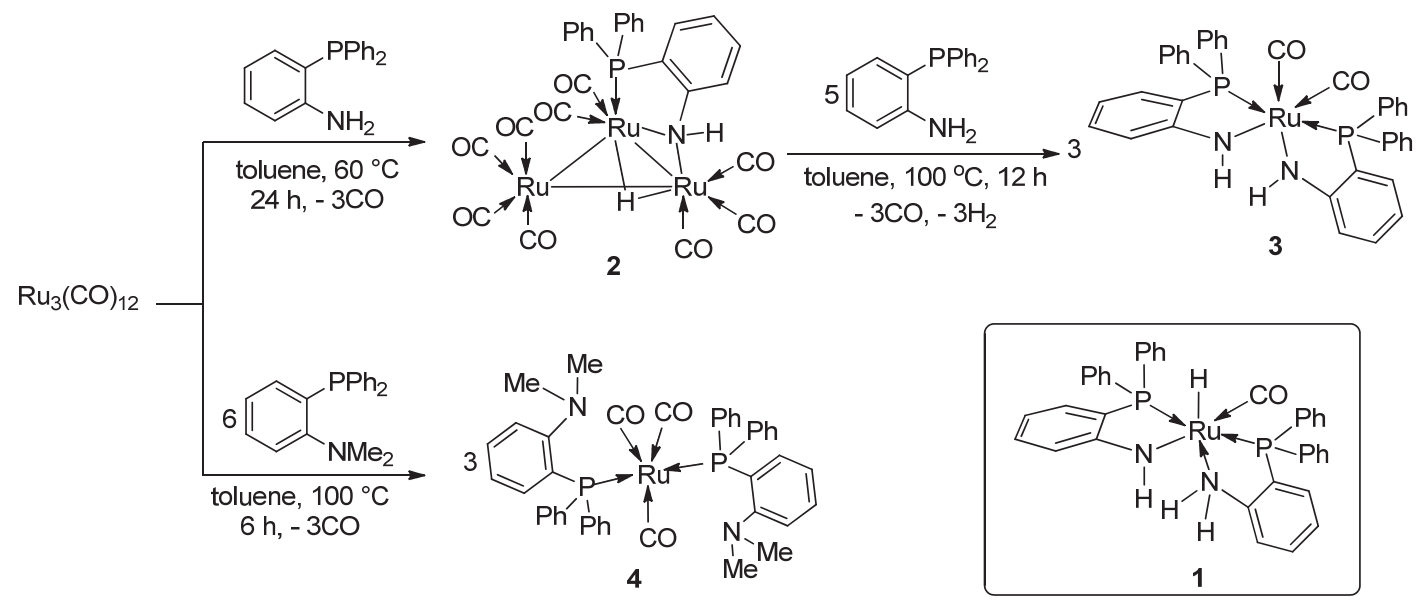

图式 2 钉化合物 1 的结构和钉化合物 $\mathbf{2} \sim \mathbf{4}$ 的合成

Scheme 2 Structure of ruthenium complex 1 and synthesis of ruthenium complexes $2 \sim 4$ 
$\mathrm{C}_{6} \mathrm{H}_{4} \mathrm{NH}_{2}$ 在甲苯溶液中于 $100{ }^{\circ} \mathrm{C}$ 反应 $12 \mathrm{~h}$, 得到化合物 $\left(o-\mathrm{PPh}_{2} \mathrm{C}_{6} \mathrm{H}_{4} \mathrm{NH}\right)_{2} \mathrm{Ru}(\mathrm{CO})_{2}(3)$. 值得注意的是, $\mathrm{Ru}_{3}(\mathrm{CO})_{12}$ 与 6 equiv. 的 $o-\mathrm{PPh}_{2} \mathrm{C}_{6} \mathrm{H}_{4} \mathrm{NH}_{2}$ 在甲苯溶液中反应也可以 得到化合物 3 . 采用甲基取代的 $o-\mathrm{PPh}_{2} \mathrm{C}_{6} \mathrm{H}_{4} \mathrm{NMe}_{2}$ 配体与 $\mathrm{Ru}_{3}(\mathrm{CO})_{12}$ 反应，仅能得到 $\left(\mu-o-\mathrm{PPh}_{2} \mathrm{C}_{6} \mathrm{H}_{4} \mathrm{NMe}_{2}\right)_{2} \mathrm{Ru}(\mathrm{CO})_{3}$ (4).

分别对化合物 2 4 开展了核磁共振和红外谱学以 及 X射线单晶衍射等结构表征, 确定了化合物的分子构 型. $\mathrm{X}$ 射线结构解析表明化合物 2 是一个三核 $\mathrm{Ru}$ 簇合物 (图 1), 每个 $\mathrm{Ru}$ 处于不同的配位结构. $\mathrm{Ru}(3)$ 与四个 $\mathrm{CO}$ 分子键联, 也同时与 $\mathrm{Ru}(1)$ 和 $\mathrm{Ru}(2)$ 键联; $\mathrm{Ru}(2)$ 键联三个 $\mathrm{CO}$ 分子的同时, 也键接一个 $\mathrm{H}$ 原子、膦胺配体中的 $\mathrm{N}$ 原子以及 $\mathrm{Ru}(3)$ 和 $\mathrm{Ru}(1) ; \mathrm{Ru}(1)$ 则与两个 $\mathrm{CO}$ 分子、一个 $\mathrm{H}$ 原子、膦胺配体中的 $\mathrm{N}$ 和 $\mathrm{P}$ 原子以及 $\mathrm{Ru}(2)$ 和 $\mathrm{Ru}(1)$ 键联. $\mathrm{Ru}(1)-\mathrm{Ru}(2) 、 \mathrm{Ru}(1)-\mathrm{Ru}(3)$ 和 $\mathrm{Ru}(2)-\mathrm{Ru}(3)$ 的键 长分别是 $0.27673(2), 0.28264(2)$ 和 $0.28296(2) \mathrm{nm}$, 这些 键长与 $\mathrm{Ru}_{3}(\mathrm{CO})_{12}$ 中的 $\mathrm{Ru}-\mathrm{Ru}$ 相当 $(0.2837 \sim 0.2859$ $\mathrm{nm})^{[7]}$, 表明 $\mathrm{Ru}(1), \mathrm{Ru}(2), \mathrm{Ru}(3)$ 之间存在着金属一金属键 作用, 其中 $\mathrm{Ru}(1)$ 与 $\mathrm{Ru}(2)$ 的作用较强. $\mathrm{Ru}(1)-\mathrm{H}(1)$ 和 $\mathrm{Ru}(2)-\mathrm{H}(1)$ 的键长都是 $0.185(3) \mathrm{nm}$, 表明形成的是 $\mu-\mathrm{H}$ 桥键. 进一步考察发现 $\mathrm{N}(1)$ 原子含有一个氢质子, 可以 推断桥 $\mu-\mathrm{H}$ 原子来自于膦胺配体原料, 显然中性的膦胺 配体与 $\mathrm{Ru}_{3}(\mathrm{CO})_{12}$ 发生了氧化还原反应 ${ }^{[8]}$. 在 ${ }^{1} \mathrm{H}$ NMR 谱 图中, $\delta-13.20$ 处的三重峰为 $\mu-\mathrm{H}$ 的共振吸收, $\delta 6.16$ 处的单峰为 $\mathrm{NH}$ 的共振吸收, $\delta 6.56 \sim 7.73$ 的多重峰为苯 环质子氢的共振吸收, 上述三组峰积分比值为 $1: 1$ : 14 ，与相应的质子组成数目吻合. 化合物 2 的 ${ }^{31} \mathrm{P} \mathrm{NMR}$ 谱仅在 $\delta 50.08$ 处显示一个单峰, 说明该化合物呈谱学 纯. ${ }^{13} \mathrm{C} \mathrm{NMR}$ 谱在 $\delta$ 171.66 213.34 呈现 9 个羰基碳的 共振吸收峰, 与晶体结构中所含 $\mathrm{CO}$ 数目一致, 表明每 个 $\mathrm{CO}$ 分子处于不同的配位环境.

化合物 3 的单晶结构如图 1 所示. 从图中可以看出, $\mathrm{R} \mathrm{u}$ 金属中心配位有两个羰基分子和两个 $\left[o-\mathrm{PPh}_{2} \mathrm{C}_{6} \mathrm{H}_{4} \mathrm{NH}\right]^{-}, \mathrm{Ru}$ 与后者呈 N,P 双齿螯合配位, 形成 稳定的 $\mathrm{RuNPC}_{2}$ 配位五元环结构. $\mathrm{Ru}$ 中心的配位数为 6 , 呈现典型的八面体配位几何构型; 两个 $\mathrm{CO}$ 分子处于 $\mathrm{Ru}$ 中心的顺式配位, 类似的是两个 $\mathrm{NH}$ 配位基, 但是两个 $o-\mathrm{PPh}_{2}$ 基处于反式配位. 由此可以看出, 2 与 $o-\mathrm{PPh}_{2} \mathrm{C}_{6} \mathrm{H}_{4} \mathrm{NH}_{2}$ 进一步发生了氧化还原反应, 可能生成 的两个 $\mathrm{H}^{-}$发生还原消除形成 $\mathrm{H}_{2}$. 化合物 3 也可以直接 由 $\mathrm{Ru}_{3}(\mathrm{CO})_{12}$ 与 $o-\mathrm{PPh}_{2} \mathrm{C}_{6} \mathrm{H}_{4} \mathrm{NH}_{2}$ 反应制得. 可以推断化 合物 2 是生成 3 的中间体, 实验结果也显示如此. 在该 化合物中, $\mathrm{Ru}$ 中心显示 +2 氧化态. ${ }^{1} \mathrm{H}$ NMR 和 ${ }^{31} \mathrm{P}$ NMR 谱分别在 $\delta 2.24$ 处显示出 $\mathrm{NH}$ 的共振吸收峰以及 $\delta 51.19$ 处显示出 $\mathrm{PPh}_{2}$ 的共振吸收峰, 说明在溶液中 $\mathbf{3}$ 以高度对
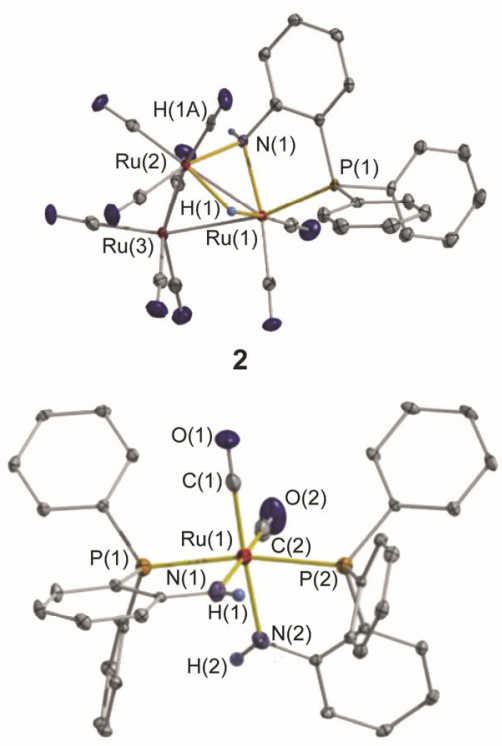

3

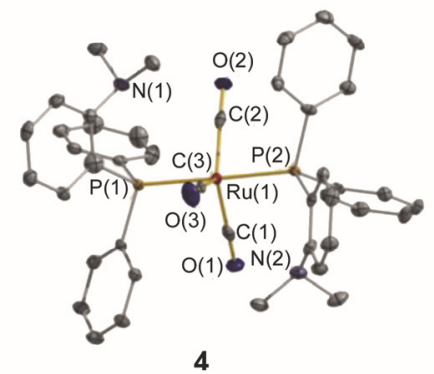

Ellipsoids probability level: $50 \%$ Most hydrogen atoms have been omitted for clarity

图 1 配合物 2 $\sim 4$ 的单晶结构图

Figure 1 X-ray crystal structure of complex $2 \sim 4$ 称的分子构型存在.

化合物 4 的单晶结构图见图 1. 在分子结构中, $o-\mathrm{PPh}_{2} \mathrm{C}_{6} \mathrm{H}_{4} \mathrm{NMe}_{2}$ 仅采用 $o-\mathrm{PPh}_{2}$ 与金属 $\mathrm{Ru}$ 配位，这一配 位方式与 $o-\mathrm{PPh}_{2} \mathrm{C}_{6} \mathrm{H}_{4} \mathrm{NH}_{2}$ 配体截然不同, 类似的实验现 象文献已有报道 ${ }^{[9]}$. 这一现象出现的原因可能是由于 $\mathrm{NMe}_{2}$ 官能团空间位阻较大, 且与 $\mathrm{Ru}(0)$ 键合能力较弱, 难以取代 CO 形成 $\mathrm{RuNPC}_{2}$ 五元环结构 ${ }^{[9,10]}$. X 射线衍射 数据显示, $\mathrm{P}(1)-\mathrm{Ru}(1)-\mathrm{P}(2)$ 为 $178.14^{\circ}$, 接近直线. 赤 道面三个 $\mathrm{CO}$ 分子以近平面型与金属中心配位 $[\mathrm{C}(1)-$ $\mathrm{Ru}(1)-\mathrm{C}(2)=116.70^{\circ}, \mathrm{C}(1)-\mathrm{Ru}(1)-\mathrm{C}(3)=124.42^{\circ}$, $\mathrm{C}(2)-\mathrm{Ru}(1)-\mathrm{C}(3)=118.93^{\circ}$. 并且, $\mathrm{P}(1)-\mathrm{Ru}(1)$ 键 $[$ 或 $\mathrm{P}(2)-\mathrm{Ru}(1)$ 键 $]$ 基本与赤道面垂直. ${ }^{1} \mathrm{H}$ NMR 和 ${ }^{31} \mathrm{P} \mathrm{NMR}$ 谱检测结果显示, 在溶液中化合物 4 以高度对称的分子 构型存在. ${ }^{13} \mathrm{C} \mathrm{NMR}$ 谱在 $\delta 45.54$ 处显示有 $\mathrm{NMe}_{2}$ 的核磁 信号，进一步验证了这一对称的分子构型.

以催化苯甲醛加氢制备苯甲醇作为模型反应，在 $100{ }^{\circ} \mathrm{C}$ 、初始 $\mathrm{H}_{2}$ 压力为 $5.0 \mathrm{MPa}$ 、反应时间为 $4 \mathrm{~h}$ 以及 $\mathrm{Ru}$ 配合物用量为 $1 \mathrm{~mol} \%$ 等反应条件下，考察了化合物 1 4 的催化加氢性能. 如表 1 所示, 催化剂 1 在该反应 
条件下实现了 $100 \%$ 的苯甲醛转化, 苯甲醇收率高达 99\% (Entry 1). 化合物 $\mathbf{2}$ 的催化活性与 $\mathrm{Ru}_{3}(\mathrm{CO})_{12}$ 相当, 取得了 $49 \%$ 的苯甲醛转化率和 $48 \%$ 的苯甲醇收率 (Entries $2 \& 5$, 表 1). 化合物 3 的结构与化合物 1 相当; 但化合物 3 没有催化活性(Entry 3, 表 1). 化合物 4 在该 反应条件下也表现出了一定的催化加氢活性(Entry 4, 苯甲醛转化率为 $27 \%$, 苯甲醇收率为 $24 \%$ ).

表 1 配合物 $1 \sim 4$ 催化苯甲醛加氢制苯甲醇的反应活性 ${ }^{a}$ Table 1 Catalytic performance of complexes $1 \sim \mathbf{4}$ in hydrogenation of benzaldehyde into benzyl alcohol

\begin{tabular}{cccc}
\hline Entry & $\mathrm{Ru}$ complex & Conv. $/ \%$ & Yield $/ \%$ \\
\hline 1 & $\mathbf{1}$ & 100 & 99 \\
2 & $\mathbf{2}$ & 49 & 48 \\
3 & $\mathbf{3}$ & 0 & - \\
4 & $\mathbf{4}$ & 27 & 24 \\
5 & $\mathrm{Ru}_{3}(\mathrm{CO})_{12}$ & 49 & 48 \\
\hline
\end{tabular}

${ }^{a}$ Reaction conditions: Benzaldehyde (0.5 mmol), Ru (1 mol\%), THF (2 mL), Temp. $=100{ }^{\circ} \mathrm{C}, p\left(\mathrm{H}_{2}\right)=5.0 \mathrm{MPa}$, Time $=4 \mathrm{~h}$.

含 $\mathrm{Ru}-\mathrm{NH}$ 功能结构的催化剂在反应过程中显示, $\mathrm{Ru}-\mathrm{H}$ 与 $\mathrm{N}-\mathrm{H}$ 有良好的协同作用 ${ }^{[2,5 a]}$. 化合物 $\mathbf{1}$ 可以 同时提供 $\mathrm{Ru}-\mathrm{H}$ 与 $\mathrm{N}-\mathrm{H}$, 因而能有效催化酯类分子加 氢 ${ }^{[\mathrm{a} a]}$, 化合物 1 取得了近乎定量的苯甲醇收率. 对比化 合物 2 与 1 的结构可以看出, 2 难以提供有效的 $\mathrm{N}-\mathrm{H}$, 但 2 有可能基于“内核加氢机理”参与催化加氢反应, 即 结构中 $\mathrm{Ru}-\mathrm{Ru}$ 键断裂生成配位空位, 以活化 $\mathrm{H}_{2}$ 分子产 生 $\mathrm{RuH}_{2}$ 活性物种, 因此表现出一定的催化活性 ${ }^{[11]}$. $\mathrm{Ru}_{3}(\mathrm{CO})_{12}$ 以及由 $o-\mathrm{PPh}_{2} \mathrm{C}_{6} \mathrm{H}_{4} \mathrm{NMe}_{2}$ 构成的化合物 4 均表 现出一定催化加氢活性, 有力验证了基于 “内核加氢机 理”参与催化加氢这一推测.

化合物 3 与 1 在催化性能上有显著差异, 有效解释 了 $\mathrm{Ru}-\mathrm{NH}$ 催化剂在宸基衍生物分子催化加氢过程中 的失活问题. 化合物 3 与 $\mathbf{1}$ 在结构上存在的差异较小, 可近似看成 1 结构中钓金属氢被羰基取代而成. 从表 1 结果可以看出, 钉金属氢被取代直接导致催化剂活性完 全丧失, 说明催化剂中 $\mathrm{Ru}$ 中心的活性基团 $\mathrm{H}^{-}$对催化性 能有着决定性影响. 化合物 3 没有催化活性, 原因是金 属中心不含有 $\mathrm{H}^{-}$, 且处于 +2 价的 $\mathrm{Ru}$ 无法进一步通过 反应形成 $\mathrm{H}^{-[5 \mathrm{a}]}$. 这一结果验证了我们在前期研究中提 出的想法, 即反应体系中甲醇分解产生 $\mathrm{CO}$, 并与金属 中心配位, 导致 $\mathrm{Ru}$ 中心无法进一步生成活性基团 $\mathrm{H}^{-}$, 造成催化剂中毒失活 ${ }^{[6]}$. 因此, 可以认为, 在反应体系 中, 低碳醇分子发生脱羰基反应是导致 $\mathrm{Ru}-\mathrm{NH}$ 功能结 构催化剂中毒失活的原因之一 ${ }^{[12]}$.

\section{2 结论}

以 $\mathrm{Ru}_{3}(\mathrm{CO})_{12}$ 和 $o-\mathrm{PPh}_{2} \mathrm{C}_{6} \mathrm{H}_{4} \mathrm{NR}_{2}(\mathrm{R}=\mathrm{H}, \mathrm{Me})$ 为原料, 合成了羰基钓配合物 $\mathbf{2} \sim \mathbf{4}$, 并采用核磁和红外谱学及 $\mathrm{X}$
射线单晶衍射等测试技术对其进行了表征. 催化苯甲醛 加氢的活性测试表明，化合物 $\mathbf{1}$ 通过 “金属配体双功能 机理”显示优良催化加氢性能; 化合物 $\mathbf{2}$ 和 $\mathbf{4}$ 可通过 “内 核加氢机理”参与催化加氢; 化合物 $\mathbf{3}$ 没有催化活性. 进 一步说明在 $\mathrm{Ru}-\mathrm{NH}$ 功能结构催化剂参与的反应体系 中, 甲醇等低碳醇分子脱羰基生成 $\mathrm{CO}$ 是导致催化剂中 毒失活的重要原因之一. 本研究有望对进一步开发合成 高效的 $\mathrm{Ru}-\mathrm{NH}$ 功能结构催化剂的设计提供指导.

\section{3 实验部分}

\section{1 仪器与试剂}

核磁共振测试在 Bruker Advance II $500 \mathrm{MHz}$ 上完 成, 以 $\mathrm{CDCl}_{3}$ 或 $\mathrm{C}_{6} \mathrm{D}_{6}$ 为氞代溶剂; 晶体学数据在 Agilent SuperNova $X$ 射线单晶衍射仪上采集; 红外光谱测试以 及 $\mathrm{C} 、 \mathrm{H} 、 \mathrm{~N}$ 元素含量的测定分别在 Nicolet FT-IR 330 型光谱仪和 Thermo Quest Italia SPA EA 1110 型元素分 析仪上完成; HRMS 采用 Q-Exactive 型液相色谱-高分辨 质谱联用仪(离子源: ESI)测定. 催化剂活性评价采用科 幂公司生产的 NSZ25-P4-T3-SS1-SV 型高压反应釜, 体 积为 $25 \mathrm{~mL}$. 反应液采用配备有氢焰离子化检测器(FID) 以及 $\mathrm{KB}-W a x$ 色谱柱 $(30 \mathrm{~m} \times 0.32 \mathrm{~mm} \times 0.33 \mu \mathrm{m})$ 的福立 GC-9790 型气相色谱仪进行分析.

甲苯、正己烷以及 $\mathrm{THF}$ 等有机溶剂购买自国药集 团上海试剂公司; $\mathrm{Ru}_{3}(\mathrm{CO})_{12}$ 、合成配体所需原料、苯甲 酫、苯甲醇以及正十二烷等购买自百灵威化学试剂公司. 配体 $o-\mathrm{PPh}_{2} \mathrm{C}_{6} \mathrm{H}_{4} \mathrm{NH}_{2}$ 以及 $o-\mathrm{PPh}_{2} \mathrm{C}_{6} \mathrm{H}_{4} \mathrm{NMe}_{2}$ 参考文献 [13]方法合成.

实验中涉及无水无氧的操作采用标准 Schlenk 技术 或在氩气氛手套箱中进行. 有机溶剂甲苯、正己烷以及 THF 等用钠丝预处理后, 在氮气气氛下用钠钾合金回流 后取用. $\mathrm{C}_{6} \mathrm{D}_{6}$ 在手套箱中用钠钾合金室温搅拌 $2 \mathrm{~d}$ 后过 滤使用; $\mathrm{CDCl}_{3}$ 用氢化钙室温搅拌 $2 \mathrm{~d}$ 并在氮气气氛下减 压蒸馏后置于手套箱中备用. 苯甲醛用氢化钙室温摚拌 2 天后在氮气气氛下蒸馏, 储存在手套箱中备用.

\section{2 实验方法}

3.2.1 配合物 $\left(\mu_{2}-2-\mathrm{PPh}_{2} \mathrm{C}_{6} \mathrm{H}_{4} \mathrm{NH}\right) \mathrm{Ru}_{3}\left(\mu_{2}-\mathrm{H}\right)(\mathrm{CO})_{9}$ (2) 的合成

在氩气氛手套箱中, 称取 $0.19 \mathrm{~g} \mathrm{Ru}_{3}(\mathrm{CO})_{12}(0.3$ $\mathrm{mmol}) 、 0.08 \mathrm{~g} o-\mathrm{PPh}_{2} \mathrm{C}_{6} \mathrm{H}_{4} \mathrm{NH}_{2}(0.3 \mathrm{mmol})$ 于装有 $30 \mathrm{~mL}$ 甲苯的 $100 \mathrm{~mL}$ 反应瓶中, 密闭后转移出手套箱, 并加 热至 $60{ }^{\circ} \mathrm{C}$. 反应 $24 \mathrm{~h}$ 后, 冷却反应液至室温. 减压浓 缩所得到的棕黄色溶液至约 $2 \mathrm{~mL}$ 后, 加入 $5 \mathrm{~mL}$ 正己烷, 迅速析出棕色沉淀. 过滤收集并用正已烷洗涤生成的沉 淀后, 减压干燥, 得到 $0.15 \mathrm{~g}$ 配合物 $\mathbf{2}$, 产率为 $62 \% .{ }^{1} \mathrm{H}$ NMR plus ${ }^{1} \mathrm{H}-{ }^{13} \mathrm{C}$ HSQC $\left(500 \mathrm{MHz}, \mathrm{C}_{6} \mathrm{D}_{6}\right) \delta:-13.20(\mathrm{~d}$, 
1H, $\left.\mu_{2}-\mathrm{H}\right), 6.16(\mathrm{~s}, 1 \mathrm{H}, \mathrm{NH}), 6.56(\mathrm{~m}), 6.80(\mathrm{~m}), 6.90(\mathrm{~s})$, $6.92(\mathrm{~s}), 6.95(\mathrm{~m}), 7.24(\mathrm{~m}), 7.73\left(\mathrm{~m}, 14 \mathrm{H}, \mathrm{C}_{6} \mathrm{H}_{4}, \mathrm{C}_{6} \mathrm{H}_{5}\right)$; ${ }^{13} \mathrm{C}$ NMR $\left(125 \mathrm{MHz}, \mathrm{C}_{6} \mathrm{D}_{6}\right) \delta: 121.4\left(\mathrm{~d}, J_{\mathrm{PC}}=43.8 \mathrm{~Hz}\right)$, $123.7\left(\mathrm{~d}, J_{\mathrm{PC}}=10.0 \mathrm{~Hz}\right), 124.0\left(\mathrm{~d}, J_{\mathrm{PC}}=5.0 \mathrm{~Hz}\right), 128.8(\mathrm{q}$, $\left.J_{\mathrm{PC}}=5.0,10.0 \mathrm{~Hz}\right), 130.0\left(\mathrm{~d}, J_{\mathrm{PC}}=2.5 \mathrm{~Hz}\right), 130.8\left(\mathrm{~d}, J_{\mathrm{PC}}=\right.$ $2.5 \mathrm{~Hz}), 131.0\left(\mathrm{~d}, J_{\mathrm{PC}}=11.3 \mathrm{~Hz}\right), 132.2(\mathrm{~s}), 132.3(\mathrm{~s})$, $133.0\left(\mathrm{~d}, J_{\mathrm{PC}}=12.5 \mathrm{~Hz}\right), 135.1(\mathrm{~s}), 135.5\left(\mathrm{~s}, \mathrm{C}_{6} \mathrm{H}_{4}, \mathrm{C}_{6} \mathrm{H}_{5}\right)$, $171.7\left(\mathrm{~d}, J_{\mathrm{PC}}=27.5 \mathrm{~Hz}, \mathrm{CO}\right), 183.6(\mathrm{~s}, \mathrm{CO}), 194.3(\mathrm{~s}, \mathrm{CO})$, $196.8\left(\mathrm{~d}, J_{\mathrm{PC}}=17.5 \mathrm{~Hz}, \mathrm{CO}\right), 198.8(\mathrm{~s}, \mathrm{CO}), 200.5(\mathrm{~s}, \mathrm{CO})$, 206.2 (d, $\left.J_{\mathrm{PC}}=6.3 \mathrm{~Hz}, \mathrm{CO}\right), 206.9$ (s, CO), 213.3 (s, CO); ${ }^{31} \mathrm{P}$ NMR (202 MHz, $\left.\mathrm{C}_{6} \mathrm{D}_{6}\right) \delta: 50.08$ (s); IR (KBr) v: 1953, 1979, 1994, 2015, 2039, $2078(\mathrm{CO}), 2961(\mathrm{NH}) \mathrm{cm}^{-1}$. Anal. calcd for $\mathrm{Ru}_{3} \mathrm{C}_{27} \mathrm{H}_{16} \mathrm{NPO}_{9}$ : C 38.94, N 1.68, H 1.94; found C 38.39, N 1.76, H 1.85. 配合物 2 (CCDC 1934516)的单晶是在手套箱中, 室温下 2 的甲苯溶液经 正己烷缓慢渗透 $4 \mathrm{~d}$ 长成.

\subsection{2 配合物 $\left(o-\mathrm{PPh}_{2} \mathrm{C}_{6} \mathrm{H}_{4} \mathrm{NH}\right)_{2} \mathrm{Ru}(\mathrm{CO})_{2}(3)$ 的合成}

在氩气氛手套箱中, 称取 $0.19 \mathrm{~g} \mathrm{Ru}_{3}(\mathrm{CO})_{12}(0.3$ $\mathrm{mmol}) 、 0.50 \mathrm{~g} o-\mathrm{PPh}_{2} \mathrm{C}_{6} \mathrm{H}_{4} \mathrm{NH}_{2}(1.8 \mathrm{mmol}$ )于装有 $40 \mathrm{~mL}$ 甲苯的 $100 \mathrm{~mL}$ 反应瓶中[或 $0.17 \mathrm{~g}$ 配合物 $2(0.2 \mathrm{mmol})$ 、 $\left.0.28 \mathrm{~g} \mathrm{o}-\mathrm{PPh}_{2} \mathrm{C}_{6} \mathrm{H}_{4} \mathrm{NH}_{2}(1.0 \mathrm{mmol})\right]$, 密闭后转移出手套 箱, 并加热至 $100{ }^{\circ} \mathrm{C}$. 反应 $12 \mathrm{~h}$ 后, 冷却反应液至室温. 减压浓缩所得到的黄色溶液至约 $2 \mathrm{~mL}$ 后, 加入 $5 \mathrm{~mL}$ 正 己烷, 析出大量黄色沉淀. 过滤收集并用正己烷洗涤生 成的沉淀后, 减压干燥, 得到 $0.50 \mathrm{~g}$ 配合物 $\mathbf{3}$, 产率为 $78 \%$ (或 $0.35 \mathrm{~g}$ 配合物 3, 产率为 $83 \%$ ). ${ }^{1} \mathrm{H}$ NMR plus ${ }^{1} \mathrm{H}-{ }^{13} \mathrm{C}$ HSQC (500 MHz, $\mathrm{CDCl}_{3}$ ) $\delta: 2.24$ (t, 2H, NH), 6.00 (m), $6.18(\mathrm{~m}), 6.82(\mathrm{t}), 6.98(\mathrm{~m}), 7.45$ (br), 7.53 (br), 7.65 (m), $7.75\left(\mathrm{~m}, 28 \mathrm{H}, \mathrm{C}_{6} \mathrm{H}_{4}, \mathrm{C}_{6} \mathrm{H}_{5}\right) ;{ }^{13} \mathrm{C}$ NMR $(125 \mathrm{MHz}$, $\left.\mathrm{CDCl}_{3}\right) \delta: 105.2\left(\mathrm{~d}, J_{\mathrm{PC}}=27.5 \mathrm{~Hz}\right), 105.5\left(\mathrm{~d}, J_{\mathrm{PC}}=27.5\right.$ $\mathrm{Hz}), 111.0\left(\mathrm{~d}, J_{\mathrm{PC}}=5.0 \mathrm{~Hz}\right), 111.0\left(\mathrm{~d}, J_{\mathrm{PC}}=5.0 \mathrm{~Hz}\right), 118.2$ $\left(\mathrm{d}, J_{\mathrm{PC}}=6.3 \mathrm{~Hz}\right), 118.3\left(\mathrm{~d}, J_{\mathrm{PC}}=6.3 \mathrm{~Hz}\right), 128.8\left(\mathrm{~d}, J_{\mathrm{PC}}=\right.$ $17.5 \mathrm{~Hz}), 128.8\left(\mathrm{~d}, J_{\mathrm{PC}}=7.5 \mathrm{~Hz}\right), 128.8\left(\mathrm{~d}, J_{\mathrm{PC}}=2.5 \mathrm{~Hz}\right)$, $129.7(\mathrm{~s}), 129.85(\mathrm{~s}), 130.0$ (s), $130.4\left(\mathrm{~d}, J_{\mathrm{PC}}=7.5 \mathrm{~Hz}\right)$, $132.1(\mathrm{~s}), 132.6\left(\mathrm{~d}, J_{\mathrm{PC}}=6.3 \mathrm{~Hz}\right), 132.6\left(\mathrm{~d}, J_{\mathrm{PC}}=6.3 \mathrm{~Hz}\right)$, $133.0\left(\mathrm{~d}, J_{\mathrm{PC}}=5.0 \mathrm{~Hz}\right), 133.0\left(\mathrm{~d}, J_{\mathrm{PC}}=5.0 \mathrm{~Hz}\right), 133.5(\mathrm{~s})$, $134.1\left(\mathrm{~d}, J_{\mathrm{PC}}=25.0 \mathrm{~Hz}\right), 134.3\left(\mathrm{~d}, J_{\mathrm{PC}}=25.0 \mathrm{~Hz}\right), 169.4(\mathrm{~d}$, $\left.J_{\mathrm{PC}}=16.3 \mathrm{~Hz}, \mathrm{C}_{6} \mathrm{H}_{4}, \mathrm{C}_{6} \mathrm{H}_{5}\right), 197.0\left(\mathrm{~d}, J_{\mathrm{PC}}=10.0 \mathrm{~Hz}, \mathrm{CO}\right)$; ${ }^{31} \mathrm{P}$ NMR (202 MHz, $\mathrm{CDCl}_{3}$ ) $\delta: 51.19$ (s); IR (KBr) $v$ : 1964, 2032 (CO), 2960 (NH) cm ${ }^{-1}$; HRMS (ESI) calcd for $\mathrm{RuC}_{38} \mathrm{H}_{31} \mathrm{~N}_{2} \mathrm{P}_{2} \mathrm{O}_{2} \quad[\mathrm{M}+\mathrm{H}]^{+}$711.0904, found 711.0913. Anal. calcd for $\mathrm{RuC}_{38} \mathrm{H}_{30} \mathrm{~N}_{2} \mathrm{P}_{2} \mathrm{O}_{2}\left(M_{\mathrm{r}}=709.7\right)$ : C 64.31, N 3.95, H 4.26; found C 64.32, N 3.82, H 4.40. 配合物 3 (CCDC 1934517)的单晶是在手套箱中, 室温下 3 的甲苯 溶液经正已烷缓慢渗透 $4 \mathrm{~d}$ 长成.
3.2.3 配合物 $\left(\mu_{1}-o-\mathrm{PPh}_{2} \mathrm{C}_{6} \mathrm{H}_{4} \mathrm{NMe}_{2}\right)_{2} \mathrm{Ru}(\mathrm{CO})_{3}$ (4) 的合 成

在氩气氛手套箱中, 称取 $0.13 \mathrm{~g} \mathrm{Ru}_{3}(\mathrm{CO})_{12}(0.2$ $\mathrm{mmol}) 、 0.37 \mathrm{~g} o-\mathrm{PPh}_{2} \mathrm{C}_{6} \mathrm{H}_{4} \mathrm{NMe}_{2}(1.2 \mathrm{mmol})$ 于装有 $30 \mathrm{~mL}$ 甲苯的 $100 \mathrm{~mL}$ 反应瓶中, 密闭后转移出手套箱, 并加 热至 $100{ }^{\circ} \mathrm{C}$. 反应 $24 \mathrm{~h}$ 后, 冷却反应液至室温. 减压浓 缩得到的黄色溶液至约 $2 \mathrm{~mL}$ 后, 加入 $5 \mathrm{~mL}$ 正已烷, 迅 速析出大量乳黄色沉淀. 过滤收集并用正己烷洗涤生成 的沉淀后, 减压干燥, 得到 $0.35 \mathrm{~g}$ 配合物 $\mathbf{4}$, 产率为 71\%. ${ }^{1} \mathrm{H}$ NMR (500 MHz, $\left.\mathrm{C}_{6} \mathrm{D}_{6}\right) \delta: 2.37\left(\mathrm{~s}, 12 \mathrm{H}, \mathrm{CH}_{3}\right)$, $6.84(\mathrm{t}), 7.03(\mathrm{~m}), 7.09(\mathrm{t}), 7.11(\mathrm{~s}), 7.16(\mathrm{~s}), 7.17(\mathrm{~s}), 7.44$ (m), $7.91\left(\mathrm{~m}, 28 \mathrm{H}, \mathrm{C}_{6} \mathrm{H}_{4}, \mathrm{C}_{6} \mathrm{H}_{5}\right) ;{ }^{13} \mathrm{C}$ NMR $(125 \mathrm{MHz}$, $\left.\mathrm{C}_{6} \mathrm{D}_{6}\right) \delta: 45.5\left(\mathrm{~s}, \mathrm{CH}_{3}\right), 125.0\left(\mathrm{t}, J_{\mathrm{PC}}=5.0 \mathrm{~Hz}\right), 125.3(\mathrm{t}$, $\left.J_{\mathrm{PC}}=2.5 \mathrm{~Hz}\right), 129.2(\mathrm{~s}), 131.6(\mathrm{~s}), 134.2\left(\mathrm{t}, J_{\mathrm{PC}}=6.3 \mathrm{~Hz}\right)$, $134.9\left(\mathrm{t}, J_{\mathrm{PC}}=25.0 \mathrm{~Hz}\right), 137.8\left(\mathrm{t}, J_{\mathrm{PC}}=22.5 \mathrm{~Hz}\right), 158.4(\mathrm{t}$, $\left.J_{\mathrm{PC}}=3.8 \mathrm{~Hz}, \mathrm{C}_{6} \mathrm{H}_{4}, \mathrm{C}_{6} \mathrm{H}_{5}\right), 209.7\left(\mathrm{t}, J_{\mathrm{PC}}=16.3 \mathrm{~Hz}, \mathrm{CO}\right) ;{ }^{31} \mathrm{P}$ NMR (202 MHz, $\left.\mathrm{C}_{6} \mathrm{D}_{6}\right) \delta: 47.96$ (s); IR (KBr) v: 1878, 1894 (CO) $\mathrm{cm}^{-1}$; HRMS (ESI) calcd for $\mathrm{RuC}_{43} \mathrm{H}_{41} \mathrm{~N}_{2} \mathrm{P}_{2} \mathrm{O}_{3}$ $[\mathrm{M}+\mathrm{H}]^{+}$797.1635, found 797.1626. Anal. calcd for $\mathrm{RuC}_{43} \mathrm{H}_{40} \mathrm{~N}_{2} \mathrm{P}_{2} \mathrm{O}_{3}$ : C 64.89, N 3.52, H 5.07; found C 64.53, N 3.38, H 5.25. 配合物 4 (CCDC 1934518) 的单晶是在手 套箱中, 在 $-20{ }^{\circ} \mathrm{C}$ 条件下 4 的甲苯溶液经正已烷缓慢 渗透 $2 \mathrm{~d}$ 长成.

\subsection{4 钓配合物的活性评价过程}

在手套箱中配制好钓配合物以及苯甲醛的 THF 溶 液, 吸入注射器中后转移出手套箱. 紧接着, 将反应液 快速注射进 $\mathrm{H}_{2}$ 保护的反应釜釜体中, 充 $\mathrm{H}_{2}$ 压力至 5 $\mathrm{MPa}$ 后，将釜体置于加热装置中按指定条件加热反应. 反应完成后，快速冷却釜体并排去釜中剩余的氢气．最 后，在反应液中加入一定量正十二烷，混合均匀后用色 谱检测. 色谱检测所用参数如下: $\mathrm{N}_{2}$ 为载气(流速为 25 $\mathrm{mL} / \mathrm{min}$ ), 气化室和 FID 检测器温度均为 $230{ }^{\circ} \mathrm{C}$, 程序 升温过程为 $50{ }^{\circ} \mathrm{C}$ 维持 $5 \mathrm{~min}$ 后以 $10{ }^{\circ} \mathrm{C} / \mathrm{min}$ 升高温度 至 $200{ }^{\circ} \mathrm{C}$ 并维持 $10 \mathrm{~min}$. 根据所得谱图中产物、原料以 及正十二烷的峰面积进行转化率和产率的计算.

辅助材料(Supporting Information) 配合物 $2 \sim 4$ 的 ${ }^{1} \mathrm{H}$ $\mathrm{NMR},{ }^{13} \mathrm{C}$ NMR 谱图及晶体结构数据. 这些材料可以免 费从本刊网站(http://sioc-journal.cn/)上下载.

\section{References}

[1] (a) Dub, P. A.; Ikariya, T. ACS Catal. 2012, 2, 1718. (b) Werkmeister, S.; Junge, K.; Beller, M. Org. Process Res. Dev. 2014, 18, 289.

(c) Pritchard, J.; Filonenko, G. A.; van Putten, R.; Hensen, E. J. M.; Pidko, E. A. Chem. Soc. Rev. 2015, 44, 3808.

(d) Zhang, Y. W.; Chen, Y. L.; Fang, X. L.; Yuan, Y. Z.; Zhu, H. P. Chin. J. Org. Chem. 2017, 37, 2275 (in Chinese). 
(张亦伟, 陈艺林, 方霄龙, 袁友珠, 朱红平, 有机化学, 2017, 37, 2275.)

[2] (a) Sandoval, C. A.; Ohkuma, T.; Muñiz, K.; Noyori, R. J. Am. Chem. Soc. 2003, 125, 13490.

(b) Saudan, L. A.; Saudan, C. M.; Debieux, C.; Wyss, P. Angew. Chem., Int. Ed. 2007, 46, 7473.

(c) Han, Z.; Rong, L.; Wu, J.; Zhang, L.; Wang, Z.; Ding, K. Angew. Chem., Int. Ed. 2012, 51, 13041.

(d) Zhao, B.; Han. Z.; Ding. K. Angew. Chem., Int. Ed. 2013, 52, 4744 .

(e) Tan, X.; Wang, Y.; Liu, Y.; Wang, F.; Shi, L.; Lee, K. H.; Lin, Z.; Lv, H.; Zhang, X. Org. Lett. 2015, 17, 454

[3] (a) Zhang, J.; Leitus, G.; Ben-David, Y.; Milstein, D. Angew. Chem., Int. Ed. 2006, 45, 1113.

(b) Balaraman, E.; Gunanathan, C.; Zhang, J.; Shimon, L. J. W.; Milstein, D. Nat. Chem. 2011, 3, 609.

(c) Gunanathan, C.; Milstein, D. Acc. Chem. Res. 2011, 44, 588.

(d) Li, W.; Xie, J. H.; Yuan, M. L.; Zhou, Q. L. Green Chem. 2014, 16, 4081 .

[4] (a) Hanton, M. J.; Tin, S.; Boardman, B. J.; Miller, P. J. Mol. Catal. A: Chem. 2011, 346, 70.

(b) vom Stein, T.; Meuresch, M.; Limper, D.; Schmitz, M.; Hölscher, M.; Coetzee, J.; Cole-Hamilton, D. J.; Klankermayer, J.; Leitner, W. J. Am. Chem. Soc. 2014, 136, 13217.

[5] (a) Fang, X.; Zhang, C.; Chen, J.; Zhu, H.; Yuan, Y. RSC Adv. 2016, 6,45512 .

(b) Fang, X.; Sun, M.; Zheng, J.; Li, B.; Ye, L.; Wang, X.; Cao, Z.; Zhu, H.; Yuan, Y. Sci. Rep. 2017, 7, 3961.

(c) Fang, X.; Li, B.; Zheng, J.; Wang, X.; Zhu, H.; Yuan, Y. Dalton
Trans. 2019, 48, 2290

[6] Fang, X.; Duan, N.; Zhang, M.; Zhang, C.; Liu, R.; Zhu, H. P. Chin J. Org. Chem. 2019, 39, 1450 (in Chinese).

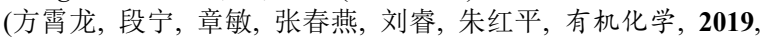
39, 1450.)

[7] Mason, R.; Rae, A. I. M. J. Chem. Soc. A 1968, 778.

[8] (a) Deeming, A. J.; Martin, C. M. Angew. Chem., Int. Ed. 1998, 37, 1691.

(b) Hanif, K. M.; Hursthouse, M. B.; Kabir, S. E.; Malik, K. M. A.; Rosenberg, E. J. Organomet. Chem. 1999, 580, 60.

(c) Tan, X.; Li, B.; Xu, S.; Song, H.; Wang, B. Organometallics 2011, 30, 2308.

[9] Gong, P.; Wu, B.; Liu, S.; Wang, D. Chin. J. Inorg. Chem. 2004, 20, 37 (in Chinese).

(宫培军, 吴秉芳, 刘树堂, 王丁泽, 无机化学学报, 2004, 20, 37.)

[10] Teunissen, H. T.; Elsevier, C. J. Chem. Commun. 1997, 667.

[11] (a) El-Amouri, H.; Bahsoun, A. A.; Fischer, J.; Osborn, J. A. Angew. Chem., Int. Ed. 1987, 26, 1169.

(b) Holland, G. F.; Ellis, D. E.; Tyler, D. R.; Gray, H. B.; Trogler, W. C. J. Am. Chem. Soc. 1987, 109, 4276.

[12] (a) Van der Sluys, L. S.; Kubas, G. J.; Caulton, K. G. Organometallics 1991, 10, 1033.

(b) Chen, Y. Z.; Chan, W. C.; Lau, C. P.; Chu, H. S.; Lee, H. L.; Jia, G. Organometallics 1997, 16, 1241.

[13] (a) Herd, O.; Heßler, A.; Hingst, M.; Tepper, M.; Stelzer, O. J. Organomet. Chem. 1996, 522, 69.

(b) Richard, V.; Ipouck, M.; Mérel, D. S.; Gaillard, S.; Whitby, R. J.; Witulski, B.; Renaud, J. L. Chem. Commun. 2014, 50, 593.

(Zhao, C.) 